\title{
Producción espacial y temporal de hojarasca del manglar en la laguna Barra de Navidad, Jalisco, México
}

\author{
Alma J. Mendoza-Morales ${ }^{1,2}$, Gaspar González-Sansón ${ }^{1,3^{*}}$ \& Consuelo Aguilar-Betancourt ${ }^{1,3}$ \\ 1. Departamento de Estudios para el Desarrollo Sustentable de Zonas Costeras. Av. Gómez Farías No. 82, San Patricio \\ Melaque, Jalisco, México. CP 48980; almajocelynmendoza@gmail.com, gaspargonzalez2001@yahoo.es, \\ coquiaguilar06@yahoo.es \\ 2. Doctorado en Biosistemática, Ecología y Manejo de Recursos Naturales y Agrícolas (BEMARENA) de la Universidad \\ de Guadalajara. \\ 3. Canadian Rivers Institute,100 Tucker Park Rd, Saint John, NB E2L 4A6, Canada. \\ * Correspondencia
}

Recibido 14-V-2015. Corregido 20-IX-2015. Aceptado 22-X-2015.

\begin{abstract}
Spatial and temporal mangrove litter production in Barra de Navidad lagoon, Jalisco, México. Barra de Navidad lagoon is a coastal wetland of international importance (Ramsar site) and it is included among the 81 Mexican mangrove priority sites. One of the most valued characteristics of this lagoon is the presence of mangrove forest in a good conservation state. The goal of our research was the measurement of mangrove litter production and environmental factors influencing its dynamics. The mangrove area was divided into seven zones and litterfall was monthly sampled from November 2011 to October 2012 using $0.25 \mathrm{~m}^{2}$ square collectors made with mosquito mesh $(1 \mathrm{~mm})$ and positioned at $1.3 \mathrm{~m}$ above the ground. Abiotic variables of the interstitial water were measured simultaneously at each zone in permanent plots using a multi-parameter probe, YSI-556-M. Total mean value of litterfall production, weighted by zone surface, was $19.12 \pm 1.23 \mathrm{gPS} / \mathrm{m}^{2} . \mathrm{mo}$ (2.29 t/ha.year). This low productivity is a consequence of the region's dry climate and low tide range. The species Avicennia germinans and Laguncularia racemosa produced more than $80 \%$ of total litterfall, while Rhizophora mangle contributed only $16 \%$ and Conocarpus erectus $<4 \%$. A significant correlation between litterfall production and abiotic variables was found (e.g. salinity and interstitial water depth). We concluded that there are significant spatial variations in soil abiotic variables which are correlated with differences in mangrove species composition, and produce, together with the life cycles stages of those species, significant variations in the quantity and composition of litterfall. Future research will be focused on quantifying spatial variations in forest structure and their relationship with litterfall production. Rev. Biol. Trop. 64 (1): 259-273. Epub 2016 March 01.
\end{abstract}

Key words: litterfall, mangrove, coastal wetlands, Mexico, Eastern Tropical Pacific.

Los manglares se encuentran entre los ecosistemas más importantes de las zonas costeras; ellos contribuyen al flujo de energía entre la tierra y el mar y suministran importantes servicios ecológicos (Aburto-Oropeza et al., 2008). Una característica fundamental de los manglares es su alta producción primaria y el aporte significativo que hacen los mismos a los presupuestos de carbono en la zona costera (Bouillon et al., 2008). El indicador más utilizado de la productividad de los manglares es la cantidad de hojarasca producida por unidad de área y de tiempo (Kristensen et al., 2008). Varios autores han coincidido en señalar que la hojarasca constituye alrededor de un $30 \%$ de la producción aérea global de los manglares (Alongi et al., 2005; Bouillon et al., 2008). Por otra parte, la hojarasca es útil para examinar los patrones reproductivos anuales y la cantidad de materia orgánica que está potencialmente disponible para descomposición y exportación (Alongi, 2002).

En México, existen varias investigaciones sobre la producción de hojarasca de manglar, 
pero estas se concentran en las regiones donde este tipo de vegetación representa un porcentaje mayor con respecto al total del país, como son Yucatán (Agraz et al., 2011); Golfo de México (Barreiro-Güemes, 1999; Aké-Castillo, 2006; Utrera \& Moreno, 2008; Agraz et al., 2011; Coronado-Molina et al., 2012) y el Pacífico noroccidental (Flores-Verdugo et al., 1990; Arreola et al., 2004; López \& Ezcurra, 2012). Por otra parte, la región del Pacífico central, que abarca las costas de los estados de Jalisco, Colima y Michoacán, responde sólo por el 0.9 $\%$ de la superficie total cubierta por manglares en la república mexicana y no fue posible encontrar algún trabajo sobre la dinámica de producción de hojarasca de manglar en sitios de esta región.

La laguna de Barra de Navidad es un humedal de importancia internacional (sitio RAMSAR desde 2008), está incluida entre los 81 sitios prioritarios de manglar de México (CONABIO, 2009) y es el principal cuerpo de agua litoral (humedal costero) de la Bahía de Navidad y en general, de la costa sur de Jalisco en el Pacífico central mexicano. Por su extensión, es el tercer cuerpo de agua natural más grande de la costa del Estado de Jalisco. Una de las características de mayor valor de la laguna, es la presencia de bosques de mangle en buen estado de conservación (González-Sansón et al., 2014), por lo que este sitio ofrece una oportunidad para investigar la dinámica de producción de hojarasca del manglar y los factores ambientales que pueden influir en la misma, en una región de México donde este aspecto no ha sido investigado previamente.

En una revisión a escala mundial, Bouillon et al. (2008) encontraron que la cantidad de hojarasca producida anualmente por unidad de área varía en función de la latitud, y es mayor en las latitudes más bajas. Una relación similar, fue demostrada para la región noroccidental de México por López-Portillo \& Ezcurra (2012). Sin embargo, existe una variabilidad muy grande en los valores registrados para cada intervalo latitudinal (Bouillon et al., 2008), la cual ha sido atribuida a la diversidad de las características ambientales locales (Saenger \&
Snedaker, 1993). Estas incluyen factores como la salinidad (Bunt, 1995; Day et al., 1996; Utrera \& Moreno, 2008; Coronado-Molina et al., 2012), el contenido de nutrientes (Lanza, 1994; Coronado-Molina et al., 2012; Adame et al., 2013), las mareas (Lugo \& Snedaker, 1974) y los niveles de inundación (Agraz et al., 2011) entre otros. Por otra parte, los ciclos vitales de las especies que integran el manglar varían como resultado de una combinación muy compleja de factores ambientales abióticos, fisiología de los árboles y otros aspectos ecológicos como la polinización y la dispersión de los propágulos (Leach \& Burgin, 1985).

De acuerdo con los antecedentes planteados anteriormente, la hipótesis que se sometió a consideración con esta investigación, es que en el manglar de la laguna Barra de Navidad, existen variaciones espaciales significativas en las variables abióticas del sustrato que se correlacionan con diferencias en la composición por especies del manglar y que, junto con las etapas de los ciclos vitales de esas especies, generan variaciones significativas en la cantidad y composición de la hojarasca producida. En consecuencia, se definieron tres objetivos principales: a) medir mensualmente la caída de hojarasca y su composición, en diferentes zonas del manglar de la laguna; b) medir mensualmente variables abióticas seleccionadas del agua intersticial en esas zonas y c) medir la correlación entre las variables abióticas y la caída de hojarasca clasificada por especies y componentes.

\section{MATERIALES Y MÉTODOS}

Área de estudio: La laguna Barra de Navidad se localiza en los $19^{\circ} 11^{\prime} 25^{\prime \prime} \mathrm{N}$ 104³9'53” W, y pertenece al municipio de Cihuatlán, Jalisco (Fig. 1). Es un sistema estuarino que mantiene comunicación permanente con el mar por medio de una boca que mide 98 $\mathrm{m}$ de ancho, su profundidad oscila entre 1 y $3 \mathrm{~m}$. El espejo de agua de la laguna es de aproximadamente $3.76 \mathrm{~km}^{2}$ y su longitud máxima es 3.5 $\mathrm{km}$, con una anchura de $1.5 \mathrm{~km}$. Esta laguna fue proclamada como sitio Ramsar el 2 de febrero 


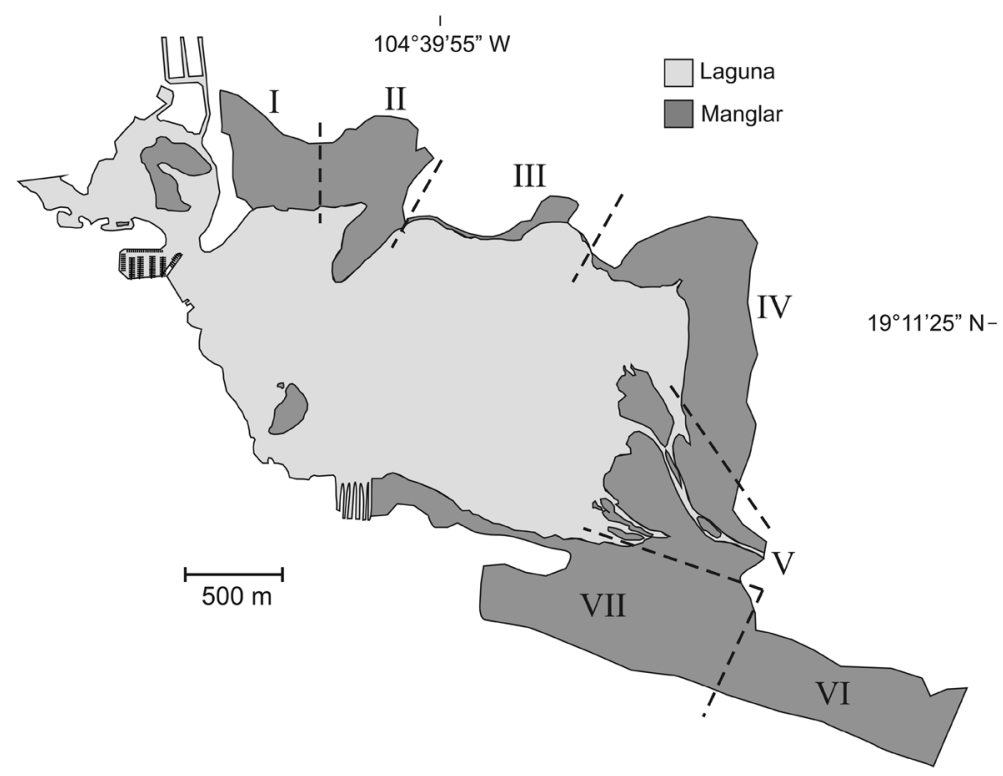

Fig. 1. Zonas del manglar delimitadas en la laguna Barra de Navidad, México.

Fig. 1. Mangrove zones delimited in Barra de Navidad lagoon, Mexico.

del 2008 (González-Sansón et al., 2014). El tipo de mareas en la zona es semidiurna mixta (dos pleamares y dos bajamares de diferentes magnitudes) con una amplitud media de unos $70 \mathrm{~cm}$ entre bajamar y pleamar. Los aportes de agua dulce a la laguna provienen principalmente del río Arroyo Seco y de un canal artificial de conexión con el río Marabasco, esencialmente durante la temporada de lluvias (Silva-Bátiz, 2012), la cual se extiende entre junio y octubre. Tomando como referencia las categorías identificadas en el Sistema de Venecia (Anónimo, 1958), la laguna es euhalina, con salinidad promedio entre 30-40, la mayor parte del tiempo, aunque en determinados periodos cortos, puede tener características mixopolihalinas con una salinidad media de 18-30 (González-Sansón et al., 2014). La vegetación de los márgenes de la laguna esta compuesta principalmente por cuatro especies de mangle: negro (Avicennia germinans L.), blanco (Laguncularia racemosa L.), rojo (Rhizophora mangle L.) y botoncillo (Conocarpus erectus L.).

Muestreo y análisis de datos: El manglar fue dividido en siete zonas, para investigar la existencia de variaciones espaciales en la caída de hojarasca y en los valores de variables abióticas (Fig. 1). Las características de esas zonas se describen a continuación:

- Zona I $\left(0.208 \mathrm{~km}^{2}\right)$. Está localizada en el margen noroccidental de la laguna y está fuertemente influenciada por las actividades antrópicas provenientes del poblado de Barra de Navidad (4 500 habitantes), y de las instalaciones hoteleras (100 habitaciones) que incluyen una marina. El flujo de agua marina hacia el manglar ha sido limitado por rellenos artificiales. La especie dominante es $A$. germinans, con árboles de 4 a $5 \mathrm{~m}$ de altura.

- Zona II $\left(0.286 \mathrm{~km}^{2}\right)$ : Es similar a la anterior, pero presenta en su borde norte un área inundada de agua dulce que en épocas de lluvias propicia escurrimiento a la laguna y el flujo de agua marina hacia el manglar no ha sido limitado por estructuras artificiales. Domina A. germinans y en menor grado, $R$. mangle. La altura media de los árboles es de $4 \mathrm{~m}$.

- Zona III $\left(0.052 \mathrm{~km}^{2}\right)$ : Incluye solamente una franja estrecha de manglar debido a la existencia de una pequeña colina cerca de 
la orilla. Vegetación mixta de $R$. mangle, A. germinans y L. racemosa. Arboles no mayores a los $5 \mathrm{~m}$ de altura.

- Zona IV $\left(0.498 \mathrm{~km}^{2}\right)$ : Corresponde con la mayor parte del delta antiguo del río Arroyo Seco y se ubica dentro de terrenos utilizados para el pastoreo de ganado. Amplio dominio de $A$. germinans. Los árboles son mayores que en las zonas anteriores, alcanzando unos $7 \mathrm{~m}$ de altura.

- Zona V $\left(0.526 \mathrm{~km}^{2}\right)$ : Corresponde con la entrada del canal artificial que trae agua desde el río Marabasco. Recibe la descarga del río Arroyo Seco en época de lluvias. Es un sitio de azolvamiento intenso donde el manglar ha invadido el espejo de agua. Domina A. germinans, pero hay una cantidad importante de L. racemosa. Arboles de alrededor de $6 \mathrm{~m}$ de altura.

- Zona VI $\left(0.465 \mathrm{~km}^{2}\right)$ : Es la zona con menor influencia marina. Para este trabajo se consideró sólo la porción más cercana a la laguna, que está al oeste de la carretera de entrada a Isla de Navidad. L. racemosa domina ampliamente. Tamaño de los árboles comparables a los de la zona IV con unos $7 \mathrm{~m}$ de altura.

- Zona VII $\left(0.783 \mathrm{~km}^{2}\right)$ : Una parte pequeña al norte de esta zona está bajo la influencia marina, pero la mayor parte de la misma se encuentra alejada del mar. L. racemosa domina ampliamente. Árboles mucho más altos que en las zonas anteriores con alturas de $18 \mathrm{~m}$ o más.

Dentro de cada zona se establecieron tres parcelas permanentes de 0.1 ha de superficie cada una, colocadas aleatoriamente en cada zona establecida previamente. Para medir la caída de hojarasca se fijaron cinco recolectores de hojarasca ubicados de forma aleatoria en cada una de las parcelas, para un total de 15 recolectores por zona. Los recolectores se construyeron con malla de mosquitero de $1 \mathrm{~mm}$, con un área de $0.25 \mathrm{~m}^{2}$ cada uno y se montaron suspendidos a $1.30 \mathrm{~m}$ sobre el suelo del manglar.
Las recolectas de hojarasca se realizaron mensualmente a partir de noviembre 2011 a octubre 2012 y el material recolectado fue transportado al laboratorio. La hojarasca fue separada por especies en sus componentes principales: hojas, flores, frutos, y una categoría denominada otros, que incluyó ramas y fragmentos de madera. Posteriormente se secaron a $60{ }^{\circ} \mathrm{C}$ por 72 horas en una estufa Felisa FE132. Se usó una balanza analítica digital (Ohaus Adventurer pro AV264C $\pm 0.0001 \mathrm{~g}$ ) para obtener el peso seco de cada fracción. La hojarasca caída se expresó como gramos de peso seco por metro cuadrado $\left(\mathrm{gPS} / \mathrm{m}^{2}\right)$. Los valores promedios mensuales para toda la laguna, se obtuvieron al ponderar por el área de cada zona.

En cada parcela de cada zona, se enterró un tubo de pvc de $55 \mathrm{~cm}$ de largo con perforaciones laterales, para permitir la entrada del agua intersticial y la creación de un pequeño pozo donde se determinó mensualmente la profundidad de la superficie del agua intersticial infiltrada y se realizaron las mediciones de las variables abióticas (temperatura, oxigeno disuelto, $\mathrm{pH}$, salinidad) con un Sistema MultiSonda (YSI $556 \mathrm{M}$ ).

Se realizaron análisis de varianza con efectos fijos para comprobar diferencias significativas entre los valores medios por sitios de muestreo y meses de las variables abióticas. Previo a los análisis, se comprobó que los datos cumplían los supuestos de normalidad y homogeneidad de varianza, aplicando los criterios recomendados por Underwood (1997). Cuando hubo significancia, se aplicó la prueba de comparaciones múltiples de Student-Newman-Keuls entre todos los pares de medias. Los datos de hojarasca total y sus componentes no cumplieron las condiciones de normalidad y homogeneidad de varianza. No se pudo encontrar una transformación que resolviera este problema y por tanto, se utilizaron pruebas no paramétricas para examinar la significación estadística de las diferencias observadas. Los valores entre zonas y entre meses se compararon mediante la prueba Kruskal-Wallis (Zar, 2010). En los casos en que esta prueba global dio significativa, se aplicó la prueba 
no paramétrica de comparaciones múltiples de Dunn, con la corrección de Dunn-Sidak (Quinn \& Keugh, 2002). Los valores de composición de la hojarasca por zonas y meses se compararon mediante el coeficiente de concordancia de Kendall (W), utilizando la función kendall.global del programa vegan en lenguaje R (Oksanen et al., 2013). Todos los demás análisis estadísticos se realizaron mediante el programa STATISTICA 7.1 (Statsoft, 2006). En todos los casos se utilizó un nivel de significación $\alpha=0.05$.

La relación entre el total de hojarasca y sus componentes por especies (variables de respuesta) y las variables abióticas del agua intersticial (variables predictoras), se investigó mediante el análisis de redundancia basado en la distancia de Hellinger (Legendre \& Gallagher, 2001). La selección de las variables abióticas significativas se realizó automáticamente y la significación estadística se determinó mediante pruebas de permutación tipo Monte Carlo sin restricciones, con 999 permutaciones. Los gráficos conjuntos (biplots) se interpretaron según las reglas resumidas en Legendre \& Legendre (2012). Los análisis fueron realizados utilizando el programa CANOCO 4.5 para Windows (ter Braak \& Smilauer, 2002).

\section{RESULTADOS}

El valor total promedio de producción de hojarasca, al ponderar por el área de cada zona, fue de $19.12 \pm 1.23 \mathrm{gPS} / \mathrm{m}^{2}$.mes. Este valor convertido a producción total anual por hectárea equivale a $2.29 \mathrm{t} / \mathrm{ha}$.año. Las especies Avicennia germinans y Laguncularia racemosa produjeron poco más del $80 \%$ de toda la hojarasca en la laguna durante el año de estudio, mientras que la especie Rhizophora mangle sólo contribuyó con el $16 \%$, y la especie Conocarpus erectus resultó de muy poca importancia (Cuadro 1). Las hojas fueron el componente predominante, siempre por encima del $70 \%$ del total de hojarasca producido por cada especie, las flores hicieron una contribución mayor en A. germinans, mientras que los frutos se destacaron en L. racemosa. La miscelánea que conforma la categoría otros, contribuyó alrededor de una décima parte en todas las especies.

La composición de la hojarasca caída total para toda la laguna presentó variaciones mensuales que fueron diferentes para cada especie (Fig. 2). En el caso de A. germinans, el coeficiente de concordancia no fue significativo ( $\mathrm{W}=0.444, \mathrm{P}=0.176)$ lo que indica que la composición de la hojarasca varió

\section{CUADRO 1}

Valores medios ( \pm error estándar) de los componentes de la hojarasca (gPS $\left./ \mathrm{m}^{2} . \mathrm{mes}\right)$ para las cuatro especies de mangle en la laguna Barra de Navidad y porcentaje que representan los componentes dentro de cada especie

TABLE 1

Mean values ( \pm standard error) of the litterfall components $\left(\mathrm{gDW} / \mathrm{m}^{2} . \mathrm{mo}\right)$ for the four mangrove species in Barra de Navidad lagoon and percentages of each component by species

\begin{tabular}{lcccc} 
& Avicennia germinans & Laguncularia racemosa & Rhizophora mangle & Conocarpus erectus \\
Hojas & $5.59 \pm 0.24$ & $5.90 \pm 0.32$ & $2.49 \pm 0.17$ & $0.40 \pm 0.07$ \\
$\%$ & 73.8 & 73.7 & 80.9 & 82.5 \\
Flores & $0.87 \pm 0.07$ & $0.35 \pm 0.05$ & $0.19 \pm 0.02$ & $0.01 \pm 0.00$ \\
$\%$ & 11.5 & 4.4 & 6.3 & 2.3 \\
Frutos & $0.29 \pm 0.04$ & $0.67 \pm 0.11$ & $0.05 \pm 0.02$ & $0.02 \pm 0.01$ \\
$\%$ & 3.8 & 8.4 & 1.5 & 4.0 \\
Otros & $0.82 \pm 0.05$ & $1.08 \pm 0.08$ & $0.35 \pm 0.05$ & $0.05 \pm 0.01$ \\
$\%$ & 10.9 & 13.5 & 11.3 & 11.2 \\
Total & $7.56 \pm 0.29$ & $7.99 \pm 0.44$ & $3.08 \pm 0.21$ & $0.49 \pm 0.09$ \\
$\%$ & 39.5 & 41.8 & 16.1 & 2.6 \\
\hline
\end{tabular}

La última fila contiene los porcentajes que cada especie representa del total de hojarasca.

Last row shows the percentage contribution of each species to total litterfall. 


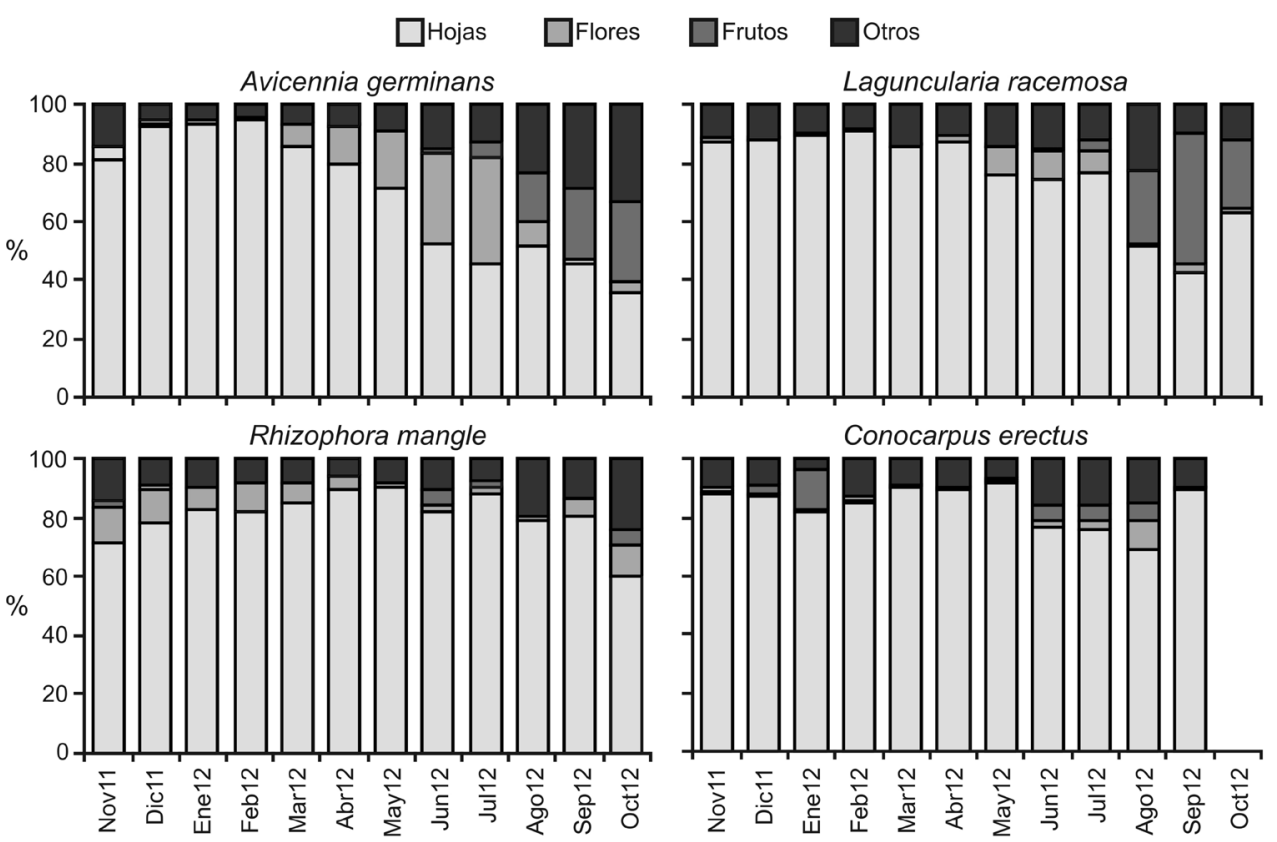

Fig. 2. Composición porcentual mensual de los componentes de la hojarasca total para cada especie.

Fig. 2. Monthly percentage composition of total litterfall components for each species.

significativamente entre meses. La proporción de hojas fue muy alta durante los meses de seca, con valores máximos entre diciembre y febrero; la proporción de flores fue mayor entre marzo y julio, con un aumento progresivo durante ese periodo, y haciéndose máxima en junio y julio, mientras que la proporción de frutos y la de otros componentes se incrementaron entre julio y octubre, con un aumento también progresivo durante ese lapso. Laguncularia racemosa presentó variaciones significativas en la composición de su hojarasca $(\mathrm{W}=0.498$, $\mathrm{P}=0.088$ ). La proporción de hojas fue muy alta durante los meses de seca, comenzando a disminuir en mayo; la proporción de flores en esta especie aumentó entre mayo y julio, mientras que la de frutos fue mayor entre agosto y octubre. $R$. mangle presentó también variaciones mensuales en los componentes de la hojarasca, que resultaron significativamente diferentes ( $\mathrm{W}=0.492, \mathrm{P}=0.092)$. Las hojas aumentaron su aporte a lo largo de la época de seca, alcanzando un máximo en mayo y presentaron una tendencia a disminuir su aporte de forma progresiva a lo largo de la época de lluvias. Las flores se encontraron entre noviembre 2011 y abril 2012, no se detectaron en cantidades apreciables entre los meses de mayo y agosto, pero volvieron a estar presentes en septiembre y octubre 2012. En el caso de $C$. erectus no se pudo verificar una diferencia significativa global en la composición de la hojarasca entre meses, porque el coeficiente de concordancia resultó significativo $(\mathrm{W}=0.689, \mathrm{P}=0.003)$.

Los valores observados de hojarasca promedio mensual recolectada para toda la laguna variaron significativamente para el total de todas las especies combinadas $(\mathrm{H}=206.01$, $\mathrm{P}<0.001)$. Al analizar las especies por separado (Fig. 3), se encontraron cambios significativos en los valores mensuales para la especie $A v i$ cennia germinans $(\mathrm{H}=55.07, \mathrm{P}<0.001)$ y para la especie Laguncularia racemosa $(\mathrm{H}=32.75$, $\mathrm{P}<0.001)$. En el caso de $A$. germinans, la prueba de comparaciones múltiples permitió identificar un valor máximo en mayo, un valor 


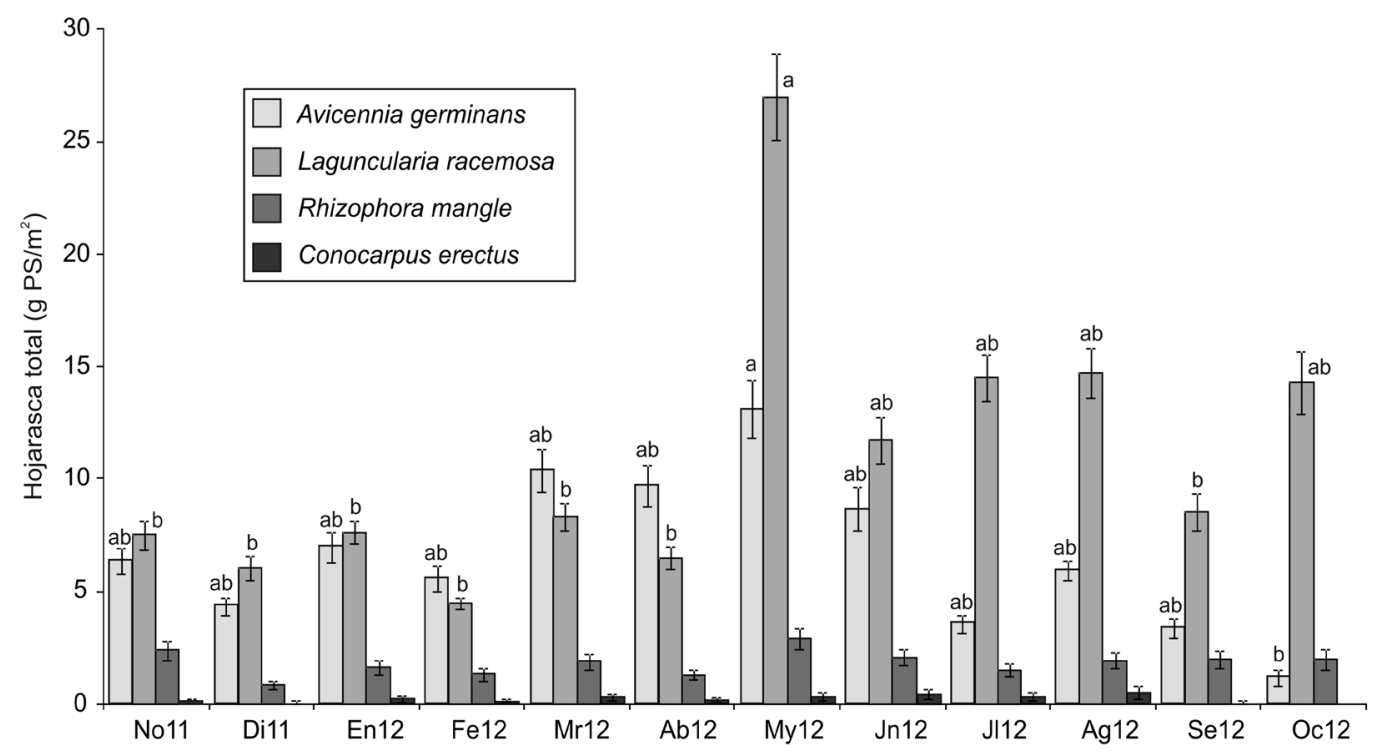

Fig. 3. Valores medios mensuales ( \pm error estándar) de la hojarasca caída $\left(\mathrm{gPS} / \mathrm{m}^{2}\right)$ clasificada por especies y ponderada por el área de las zonas. Letras iguales indican diferencia no significativa entre meses para cada especie según la prueba de comparaciones múltiples de Dunn, aplicada sólo en los casos en los que la prueba de Kruskal-Wallis arrojó diferencia significativa global.

Fig. 3. Monthly mean values ( \pm standard error) of litterfall $\left(\mathrm{gDW} / \mathrm{m}^{2}\right)$ classified by species and weighted by zone surface. Same letters indicate non significant differences among the means after Dunn's multiple comparisons test, applied only for those cases where Kruskal-Wallis test yielded a global significant difference.

mínimo en octubre y un grupo de valores intermedios para el resto de los meses. L. racemosa presentó un valor máximo también en mayo, un grupo de valores bajos en el resto de los meses de seca y septiembre, y un grupo de valores intermedios en la mayoría de los meses de lluvia. No se pudo verificar la existencia de diferencias significativas entre los valores medios mensuales de Rhizophora mangle $(\mathrm{H}=15.32, \mathrm{P}<0.186)$ y Conocarpus erectus $(\mathrm{H}=4.98, \mathrm{P}=0.932)$.

Al analizar los datos agrupados por zonas del manglar, se encontraron patrones diferentes para las distintas especies y el total combinado (Fig. 4). Para L. racemosa, la prueba global dio significativa $(\mathrm{H}=816.28, \mathrm{P}<0.001)$ y las comparaciones múltiples permitieron definir grupos homogéneos de medias, el primer grupo formado por valores altos en los sitios de muestreo VI y VII. El segundo grupo incluyó solamente al sitio $\mathrm{V}$ con un valor intermedio de producción de hojarasca y el tercer grupo estuvo formado por las zonas I a IV con valores muy bajos. En la especie $A$. germinans, la prueba global resultó significativa $(\mathrm{H}=454.71, \mathrm{P}<0.001)$; las zonas I, II, IV y $\mathrm{V}$ formaron un grupo homogéneo de valores altos, mientras que las zonas III y VII se incluyeron en un grupo de valores intermedios y la zona VI presentó una producción de hojarasca significativamente menor que en el resto de las zonas. Los valores medios por zona de $R$. mangle también fueron significativamente diferentes $(\mathrm{H}=351.36, \mathrm{P}<0.001)$. Los valores máximos se presentaron en la zona III que tuvo una producción de hojarasca significativamente mayor que el resto de las zonas, le siguió un grupo intermedio formado por las zonas II, VI y VII que presentaron valores significativamente mayores que las zonas I, IV y V. En el caso de $C$. erectus se encontró un valor significativo en la prueba global $(\mathrm{H}=235.40$, $\mathrm{P}<0.001)$. En esta especie, la zona I presentó una producción significativamente mayor que el resto de las zonas, las cuales presentaron producciones muy bajas y que no fueron diferentes entre sí. La producción de hojarasca total 


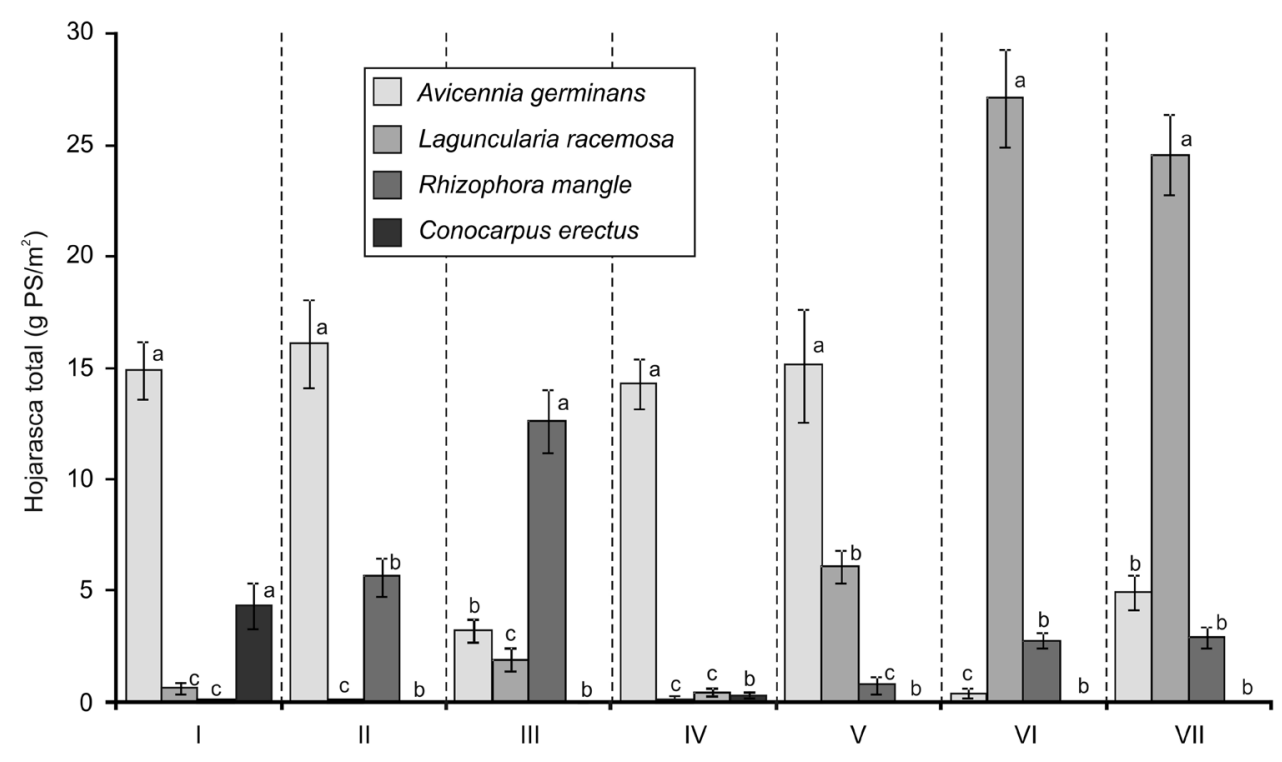

Fig. 4. Valores medios por zonas ( \pm error estándar) de la hojarasca total caída mensualmente $\left(\mathrm{gPS} / \mathrm{m}^{2}\right)$ por especies. Letras iguales indican diferencia no significativa entre zonas para cada especie según la prueba de comparaciones múltiples de Dunn.

Fig. 4. Mean values per zones ( \pm standard error) of monthly litterfall $\left(\mathrm{gDW} / \mathrm{m}^{2}\right)$ classified by species. Same letters indicate non significant differences among the means after Dunn's multiple comparisons test.

media por zonas (todas las especies sumadas) fue significativamente diferente entre zonas $(\mathrm{H}=141.89, \mathrm{P}<0.001)$. La prueba de comparaciones múltiples resultó en un grupo de producción significativamente más alta formando por las zonas VI y VII con respecto al grupo formado por el resto de las zonas.

Los valores medios de las variables abióticas presentaron diferencias entre zonas que siguieron un patrón diferente para cada variable (Cuadro 2). En el caso de la profundidad del agua intersticial, la prueba global resultó significativa $\left(\mathrm{F}_{6,570}=70.68, \mathrm{P}<0.001\right)$. La comparación de todos los pares de medias permitió comprobar que esta variable decreció significativamente entre las zonas VII, VI y IV en ese orden. El resto de las zonas debe ser combinado en un grupo homogéneo con valores menores. La salinidad resultó en general diferente entre zonas $\left(\mathrm{F}_{6,570}=169.71, \mathrm{P}<0.001\right)$. En este caso, se verificó que las zonas de la I a la $\mathrm{V}$ formaron un grupo homogéneo que presentó medias significativamente mayores que en la zona VII, cuyo valor medio fue, a su vez, mayor que en la zona VI. Un resultado bastante similar se presentó en el caso de la temperatura que arrojó diferencias significativas entre zonas $\left(\mathrm{F}_{6,570}=18.91, \mathrm{P}<0.001\right)$. De nuevo las zonas I a $\mathrm{V}$ formaron un grupo homogéneo con temperaturas medias más altas que el grupo homogéneo formado por las zonas VI y VII. En el caso del oxígeno, los valores medios resultaron bajos en general, la prueba global dio un resultado significativo $\left(\mathrm{F}_{6,570}=5.63, \mathrm{P}<0.001\right)$, pero la prueba de comparaciones múltiples no permitió definir grupos homogéneos de medias. El pH medio resultó significativamente diferente entre zonas en la prueba global $\left(\mathrm{F}_{6,570}=38.10, \mathrm{P}<0.001\right)$ y la prueba de comparaciones múltiples permitió definir que el valor medio en la zona I es significativamente mayor que en el resto de las zonas, mientras que el valor medio de esta variable en la zona VII resultó significativamente menor que en el resto de las zonas, las zonas II a VI formaron un grupo homogéneo.

En el caso de los valores medios mensuales de las variables abióticas (Cuadro 3), se encontraron diferencias significativas globales para la 
CUADRO 2

Valores medios ( \pm error estándar) de las variables físicas y químicas del agua intersticial en las zonas de estudio

TABLE 2

Mean values ( \pm standard error) of interstitial water physical and chemical variables in the zones defined for this study

\begin{tabular}{|c|c|c|c|c|c|c|c|c|c|c|}
\hline Zonas & $\begin{array}{l}\text { Profundidad } \\
\mathrm{cm}\end{array}$ & & $\begin{array}{l}\text { Salinidad } \\
\text { ups }\end{array}$ & & $\begin{array}{c}\text { Temperatura } \\
{ }^{\circ} \mathrm{C}\end{array}$ & & $\begin{array}{c}\text { Oxígeno } \\
\mathrm{mg} / \mathrm{L}\end{array}$ & & $\mathrm{pH}$ & \\
\hline I & $3.36 \pm 0.93$ & d & $38.91 \pm 2.78$ & $\mathrm{a}$ & $28.57 \pm 0.53$ & $\mathrm{a}$ & $1.12 \pm 0.24$ & $a b$ & $8.64 \pm 0.04$ & a \\
\hline II & 0 & de & $38.02 \pm 1.25$ & $\mathrm{a}$ & $26.50 \pm 0.44$ & $\mathrm{~b}$ & $0.48 \pm 0.12$ & $\mathrm{bc}$ & $8.33 \pm 0.07$ & b \\
\hline III & $0.95 \pm 0.48$ & de & $32.37 \pm 0.73$ & $\mathrm{~b}$ & $27.30 \pm 0.58$ & $a b$ & $0.69 \pm 0.13$ & $\mathrm{~b}$ & $8.29 \pm 0.05$ & b \\
\hline IV & $7.93 \pm 1.35$ & $\mathrm{c}$ & $35.13 \pm 1.86$ & $a b$ & $27.58 \pm 0.39$ & $a b$ & $0.65 \pm 0.13$ & b & $8.19 \pm 0.05$ & b \\
\hline $\mathrm{V}$ & 0 & e & $32.98 \pm 1.28$ & $a b$ & $27.03 \pm 0.28$ & $\mathrm{~b}$ & $1.46 \pm 0.29$ & $\mathrm{a}$ & $8.31 \pm 0.03$ & $\mathrm{~b}$ \\
\hline VI & $22.42 \pm 1.48$ & $\mathrm{~b}$ & $1.76 \pm 0.26$ & $\mathrm{~d}$ & $23.75 \pm 0.29$ & $\mathrm{c}$ & $0.58 \pm 0.09$ & $\mathrm{~b}$ & $8.15 \pm 0.05$ & $\mathrm{~b}$ \\
\hline VII & $28.13 \pm 0.82$ & $\mathrm{a}$ & $15.71 \pm 0.89$ & $\mathrm{c}$ & $24.48 \pm 0.22$ & $\mathrm{c}$ & $0.31 \pm 0.08$ & $\mathrm{c}$ & $7.62 \pm 0.04$ & $\mathrm{c}$ \\
\hline
\end{tabular}

Letras iguales indican diferencias no significativas entre las medias según la prueba de Student-Newman-Keuls.

Same letters indicate non significant differences among the means after the Student-Newman-Keuls test.

CUADRO 3

Valores medios mensuales ( \pm error estándar) de las variables físicas y químicas del agua intersticial

TABLE 3

Monthly mean values ( \pm standard error) of interstitial water physical and chemical variables

\begin{tabular}{lcccccccccccc}
\multicolumn{1}{c}{ mes } & $\begin{array}{c}\text { Profundidad } \\
\mathrm{cm}\end{array}$ & \multicolumn{3}{c}{$\begin{array}{c}\text { Salinidad } \\
\text { ups }\end{array}$} & & $\begin{array}{c}\text { Temperatura } \\
{ }^{\circ} \mathrm{C}\end{array}$ & $\begin{array}{c}\text { Oxígeno } \\
\mathrm{mg} / \mathrm{L}\end{array}$ & & $\mathrm{pH}$ \\
Nov. 11 & $3.83 \pm 1.34$ & $\mathrm{bc}$ & $24.78 \pm 1.80$ & $\mathrm{bcd}$ & $26.88 \pm 0.42$ & $\mathrm{ab}$ & $1.74 \pm 0.28$ & $\mathrm{a}$ & $8.19 \pm 0.06$ & $\mathrm{cdef}$ \\
Dic. 11 & $9.44 \pm 1.80$ & $\mathrm{ab}$ & $31.30 \pm 1.88$ & $\mathrm{ab}$ & $25.06 \pm 0.85$ & $\mathrm{bc}$ & $0.90 \pm 0.16$ & $\mathrm{ab}$ & $7.90 \pm 0.06$ & $\mathrm{f}$ \\
Ene. 12 & $8.95 \pm 1.88$ & $\mathrm{ab}$ & $32.80 \pm 2.07$ & $\mathrm{ab}$ & $23.45 \pm 0.22$ & $\mathrm{c}$ & $0.90 \pm 0.18$ & $\mathrm{ab}$ & $8.00 \pm 0.06$ & $\mathrm{cdef}$ \\
Feb. 12 & $10.00 \pm 2.12$ & $\mathrm{ab}$ & $28.07 \pm 2.24$ & $\mathrm{abc}$ & $22.39 \pm 0.21$ & $\mathrm{~d}$ & $1.43 \pm 0.21$ & $\mathrm{a}$ & $8.02 \pm 0.06$ & $\mathrm{cdef}$ \\
Mar. 12 & $4.51 \pm 1.75$ & $\mathrm{bc}$ & $33.08 \pm 3.93$ & $\mathrm{ab}$ & $26.45 \pm 0.73$ & $\mathrm{ab}$ & $0.02 \pm 0.00$ & $\mathrm{~b}$ & $8.75 \pm 0.05$ & $\mathrm{a}$ \\
Abr. 12 & $11.22 \pm 2.12$ & $\mathrm{ab}$ & $24.30 \pm 2.29$ & $\mathrm{bcd}$ & $26.66 \pm 0.80$ & $\mathrm{ab}$ & $1.50 \pm 0.45$ & $\mathrm{a}$ & $8.63 \pm 0.09$ & $\mathrm{ab}$ \\
May. 12 & $11.05 \pm 1.68$ & $\mathrm{ab}$ & $37.24 \pm 3.04$ & $\mathrm{a}$ & $28.20 \pm 0.20$ & $\mathrm{a}$ & $0.25 \pm 0.07$ & $\mathrm{~b}$ & $8.24 \pm 0.05$ & $\mathrm{cde}$ \\
Jun. 12 & $11.31 \pm 1.73$ & $\mathrm{ab}$ & $19.04 \pm 1.34$ & $\mathrm{~cd}$ & $28.84 \pm 0.34$ & $\mathrm{a}$ & $0.29 \pm 0.12$ & $\mathrm{~b}$ & $8.42 \pm 0.06$ & $\mathrm{bc}$ \\
Jul. 12 & $15.47 \pm 1.84$ & $\mathrm{a}$ & $27.81 \pm 1.85$ & $\mathrm{abc}$ & $27.79 \pm 0.19$ & $\mathrm{a}$ & $0.14 \pm 0.04$ & $\mathrm{~b}$ & $8.06 \pm 0.05$ & $\mathrm{cdef}$ \\
Ago. 12 & $8.41 \pm 2.06$ & $\mathrm{ab}$ & $8.88 \pm 1.89$ & $\mathrm{e}$ & $26.90 \pm 0.24$ & $\mathrm{ab}$ & $0.39 \pm 0.14$ & $\mathrm{~b}$ & $8.06 \pm 0.06$ & $\mathrm{cdef}$ \\
Sep. 12 & $15.00 \pm 3.38$ & $\mathrm{a}$ & $15.50 \pm 3.21$ & $\mathrm{de}$ & $28.48 \pm 0.51$ & $\mathrm{a}$ & $0.04 \pm 0.01$ & $\mathrm{~b}$ & $7.96 \pm 0.25$ & $\mathrm{ef}$ \\
Oct. 12 & 0 & $\mathrm{c}$ & $8.91 \pm 2.10$ & $\mathrm{e}$ & $26.93 \pm 0.34$ & $\mathrm{ab}$ & $0.91 \pm 0.41$ & $\mathrm{ab}$ & $8.30 \pm 0.09$ & $\mathrm{~cd}$ \\
\hline
\end{tabular}

Letras iguales indican diferencias no significativas entre las medias según la prueba de Student-Newman-Keuls.

Same letters indicate non significant differences among the means after the Student-Newman-Keuls test.

profundidad del agua intersticial $\left(\mathrm{F}_{6,565}=3.86\right.$, $\mathrm{P}<0.001)$, la salinidad media $\left(\mathrm{F}_{6,565}=9.80\right.$, $\mathrm{P}<0.001)$, la temperatura media $\left(\mathrm{F}_{6,565}=16.45\right.$, $\mathrm{P}<0.001)$, el oxígeno disuelto $\left(\mathrm{F}_{6,565}=9.14\right.$, $\mathrm{P}<0.001)$ y el $\mathrm{pH}\left(\mathrm{F}_{6,565}=15.78, \mathrm{P}<0.001\right)$. En ninguno de los casos fue posible determinar grupos homogéneos de medias.

El análisis de redundancia permitió definir un primer eje canónico que resultó significativo
( $\mathrm{F}=183.94, \mathrm{P}<0.002)$, explicó el $24.4 \%$ de la varianza en los datos de respuesta (hojarasca caída), 97.1\% de la relación de los componentes de la hojarasca con las variables ambientales y presentó un valor de correlación de los componentes de la hojarasca con las variables ambientales de 0.664 (Cuadro 4). El resto de los ejes canónicos tomados en conjunto explicó solo $0.07 \%$ de la varianza total de los datos de 
CUADRO 4

Análisis de redundancia basado en distancia de Hellinger

TABLE 4

Redundancy analysis based on Hellinger's distance

\begin{tabular}{lcccc}
$\quad$ Sumario de los ejes de ordenamiento & 1 & 2 & 3 & 4 \\
Valores propios & 0.244 & 0.004 & 0.003 & $<0.001$ \\
Correlación componentes-ambiente & 0.664 & 0.132 & 0.312 & 0.052 \\
$\begin{array}{l}\text { Porcentaje acumulado de varianza } \\
\quad \text { de los datos de los componentes }\end{array}$ & & & \\
$\quad$ de la relación componentes-ambiente & 24.4 & 24.8 & 25.1 & 25.1 \\
Coeficientes canónicos de las variables estandarizadas y correlaciones & 97.1 & 98.7 & 99.8 & 100 \\
inter-conjunto de las variables ambientales con los ejes canónicos & Coeficientes canónicos & Correlaciones inter-conjunto \\
Profundidad & Eje 1 & Eje 2 & Eje 1 & Eje 2 \\
Salinidad & -0.463 & 1.060 & -0.570 & 0.055 \\
Temperatura & 0.551 & 0.459 & 0.569 & 0.018 \\
Oxígeno & 0.086 & 0.433 & 0.300 & 0.064 \\
pH & 0.019 & 0.308 & 0.054 & 0.024 \\
\hline
\end{tabular}

respuesta y $2.9 \%$ de la relación de los componentes de la hojarasca con las variables ambientales. Estos resultados indican la existencia de un solo gradiente significativo explicado por el primer eje. El análisis en conjunto de los coeficientes canónicos y las correlaciones interconjunto de las variables ambientales con el primer eje, indican que el mismo expresa básicamente un gradiente de incremento en la salinidad y de disminución en la profundidad del agua intersticial infiltrada. Además, se puede considerar un componente, menos importante, de incremento del $\mathrm{pH}$ en la dirección positiva del eje. El análisis de los efectos condicionales de las variables (Cuadro 5) corrobora que las variables con efectos significativos son las mencionadas anteriormente.

El análisis del gráfico conjunto de los datos (muestras) clasificados por zonas y de las variables abióticas permite reconocer un patrón claro (Fig. 5A), con las muestras de las zonas VI y VII desplazadas hacia la izquierda sobre el eje I, indicando una correlación positiva con la profundidad del agua intersticial y negativa con la salinidad. El resto de las zonas se concentran en la porción derecha, lo que indica una correlación positiva con la salinidad y en menor grado con el $\mathrm{pH}$, por una parte y una correlación negativa con la profundidad del agua intersticial.

CUADRO 5

Efectos condicionales de las variables, en el proceso de selección por pasos automática (las variables se ordenan según la secuencia de inclusión)

TABLE 5

Conditional effects of variables in forward stepwise automatic selection procedure (variables ordered after their inclusion sequence)

\begin{tabular}{|c|c|c|c|c|c|}
\hline Variables & $\mathrm{F}$ & $P$ & $\lambda$ & Vexp & $\%$ Vexp \\
\hline Profundidad & 126.15 & 0.002 & 0.18 & 0.18 & 72 \\
\hline Salinidad & 41.82 & 0.002 & 0.06 & 0.24 & 96 \\
\hline $\mathrm{pH}$ & 7.91 & 0.002 & 0.01 & 0.25 & 100 \\
\hline Temperatura & 2.73 & 0.064 & $<0.01$ & 0.25 & 100 \\
\hline Oxígeno & 1.00 & 0.398 & $<0.01$ & 0.25 & 100 \\
\hline
\end{tabular}



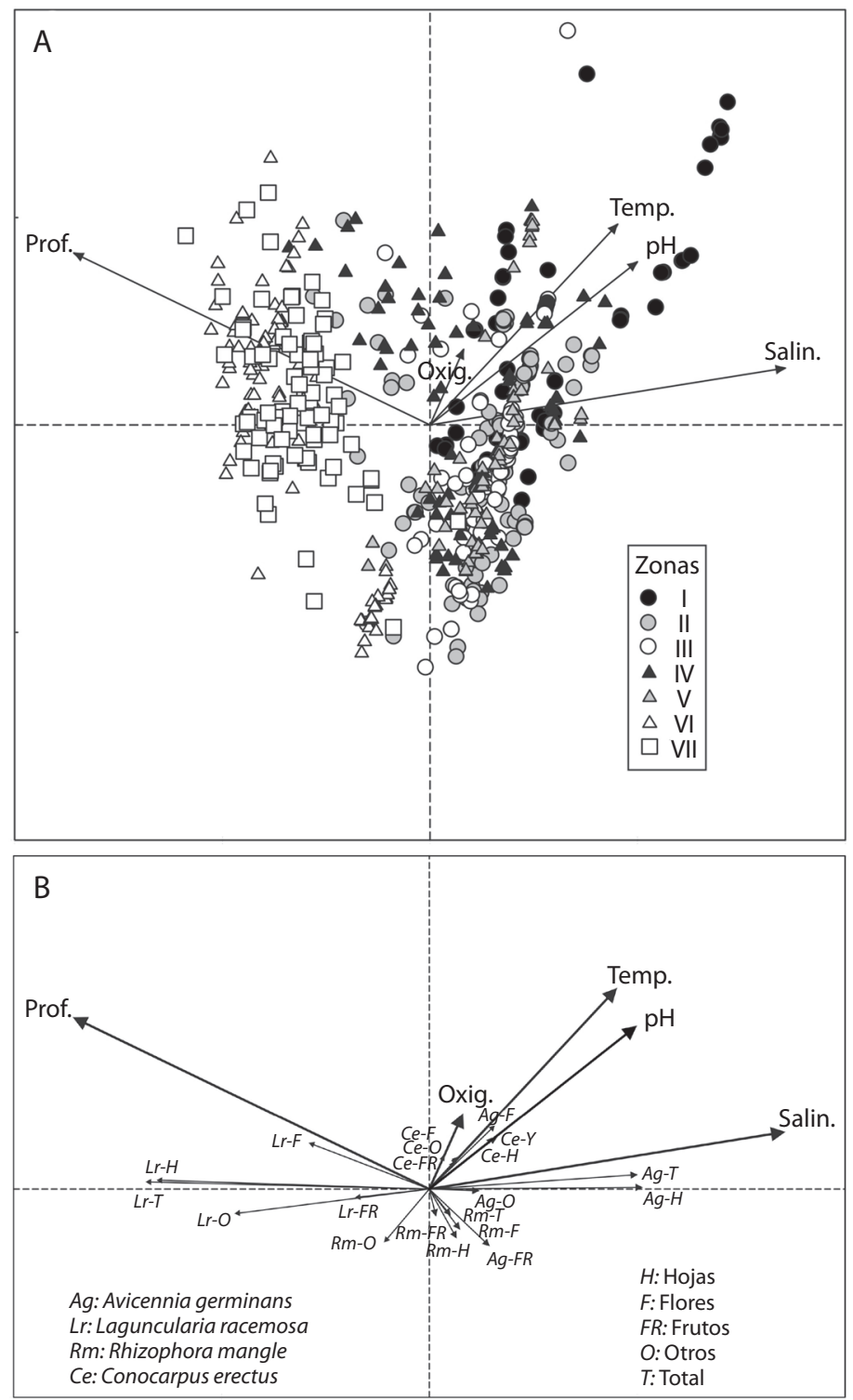

Fig. 5. Resultados del análisis de redundancia basado en distancia de Hellinger. A) Gráfico conjunto de muestras y variables abióticas. B) Gráfico conjunto de componentes de la hojarasca por especies y variables abióticas.

Fig. 5. Results of redundancy analysis based on Hellinger's distance. A) Biplot of samples and abiotic variables. B) Biplot of litterfall components by species and abiotic variables.

En el gráfico conjunto de las variables de respuesta (componentes y total de la hojarasca por especies) y las variables ambientales se expresan varias relaciones importantes (Fig. 5 B). Todos los componentes de L. racemosa presentaron una correlación negativa muy alta con el eje I, como se concluye de los ángulos muy agudos que forman las flechas que representan a las respectivas variables, con el eje. Esto implica una alta correlación de estos componentes con la profundidad del agua intersticial. En el resto de las especies, los componentes de 
la hojarasca presentaron una correlación positiva con el eje I (excepto el componente otros de $R$. mangle). Los componentes hojas, otros y total de A. germinans estuvieron altamente correlacionados con el eje y por tanto, con la salinidad. Un hecho notable, es que el componente flores de esta especie presentó una alta correlación con la temperatura y el $\mathrm{pH}$. Todos los componentes de $C$. erectus presentan alta correlación con la temperatura y el $\mathrm{pH}$, mientras que los componentes de $R$. mangle tienen una relación muy baja con cualquiera de las variables abióticas medidas.

\section{DISCUSIÓN}

Los valores de producción anual media de hojarasca obtenidos en el presente estudio se ubican cerca del límite inferior del amplio rango de valores que han sido considerados en compilaciones globales realizadas por varios autores (Flores-Verdugo, González-Farías, Zamorano, \& Ramírez-García, 1992; Saenger \& Snedakker, 1993; Utrera \& Moreno, 2008). La inmensa mayoría de los trabajos compilados han sido realizados en bosques de mangle dominados por especies de los géneros Avicennia y Rhizophora, con muy poca presencia de Laguncularia, lo que marca una diferencia notable con la presente investigación donde la mayor producción de hojarasca correspondió a las especies $L$. racemosa y $A$. germinans $(80 \%)$ con una participación mucho menor de $R$. mangle. Agraz et al. (2011) encontraron que en una zona de manglar de cuenca mixta, con dominancia de $A$. germinans y una proporción de $L$. racemosa mucho mayor que en otras zonas del mismo estudio, la producción media de hojarasca fue de 2.29 t/ha.año, comparable a los valores encontrados en nuestra investigación.

Los valores relativamente bajos de producción de hojarasca encontrados en esta laguna obedecen a que las fuentes de energía auxiliar (Mann \& Lazier, 2006) en este sistema son limitadas. Las lluvias son fuertemente estacionales y según datos históricos (González-Sansón et al., 2014), sólo hay precipitación intensa durante tres meses del año (agosto-octubre).
Este factor climático es también determinante en la presencia del bosque seco (selva baja caducifolia), formación vegetal típica de la región costera en el Estado de Jalisco (Rzedowski, 2006). Por otra parte, la amplitud de las mareas es muy reducida y este factor, que ha sido reconocido como uno de los más importantes en la modulación de la productividad del manglar (Lugo \& Snedaker, 1974; BarreiroGüemes, 1999; Arreola-Lizárraga et al., 2004; Coronado-Molina, 2012) tiene poco efecto en los manglares de este humedal costero. En concordancia con lo anterior, se debe recalcar que este sitio se encuentra en la región con menos desarrollo de manglar de México (RodríguezZúñiga et al., 2013).

En las dos especies principales que dominan ampliamente el manglar de la laguna, se pudo verificar una coincidencia notable en los periodos de floración (finales de la época de secas hasta principios de la época de lluvia) y de producción de frutos (época de lluvias avanzada). Estas mismas especies presentaron una proporción mayor de hojas en la hojarasca durante los meses de la seca, con un máximo en la caída de hojarasca en mayo. Estos resultados coinciden con lo planteado por Flores Verdugo et al. (1990) y Day et al. (1996) para otros manglares mexicanos. Según López-Portillo (1985), A. germinans podría tener una estrategia general de vida según la cual, esta especie tiende a perder más hojas durante los periodos de gran estrés hídrico, mientras que tiende a producir propágulos durante los períodos de nivel de agua alto y salinidades más bajas. Este modelo ajusta muy bien con los resultados obtenidos en nuestro trabajo para esta especie y podría ser también la explicación de los resultados similares obtenidos para L. racemosa.

Las notables variaciones entre zonas en la composición de la hojarasca son el resultado de un cambio en la composición por especies del manglar, que en última instancia responde a factores ambientales complejos que operan a escala local (Bunt, 1995; Day et al., 1996). En nuestro estudio, los dos factores que presentan una correlación mayor con la caída de hojarasca son la salinidad y la profundidad 
del agua intersticial infiltrada en los pozos de muestreo. Es importante resaltar que existe una fuerte correlación negativa entre estas variables abióticas y que las dos especies principales del manglar de la laguna responden de forma opuesta a cada variable. La especie $L$. racemosa resultó dominante en las zonas con salinidad más baja y mayor drenaje (inferido de la mayor profundidad del agua intersticial infiltrada), mientras que $A$. germinans mostró lo contrario. Estos resultados coinciden muy bien con lo encontrado por López-Portillo \& Ezcurra (2012) para estas especies.

En conclusión, nuestros resultados indican una correlación significativa entre las variables abióticas del sustrato (principalmente la salinidad y la profundidad del agua intersticial) y la cantidad de hojarasca producida por el manglar en la laguna Barra de Navidad. Por otra parte, las variaciones en los ciclos vitales de esas especies, generan cambios significativos en la composición de la hojarasca producida y contribuyen en parte a la variación espacial y temporal observada en la cantidad de hojarasca caída.

\section{AGRADECIMIENTOS}

Esta investigación fue financiada parcialmente, mediante el proyecto 05-2010-1-746 del fondo COECYTJAL-UDG 2010 y es parte del trabajo de doctorado de la primera autora, la cual reconoce con gratitud el apoyo proporcionado por CONACYT (becario: 251339). Nuestro agradecimiento a los estudiantes de la carrera de Licenciatura en Biología Marina de la Universidad de Guadalajara, que participaron como voluntarios en la investigación.

\section{RESUMEN}

La laguna de Barra de Navidad es un humedal costero de importancia internacional (sitio RAMSAR) y está incluida entre los 81 sitios prioritarios de manglar de México. Una de las características de mayor valor de la laguna, es la presencia de bosques de mangle en buen estado de conservación. El objetivo de nuestro trabajo, fue medir la producción de hojarasca del manglar y los factores ambientales que pueden influir en su dinámica.
El manglar fue dividido en siete zonas y las recolectas de hojarasca se realizaron mensualmente entre noviembre 2011 y octubre 2012, mediante recolectores cuadrados de $0.25 \mathrm{~m}^{2}$ construidos con malla de mosquitero $(1 \mathrm{~mm})$ y colocados a $1.3 \mathrm{~m}$ del suelo. De forma simultánea, se midieron varias variables abióticas en el agua intersticial recolectada en pozos permanentes en cada zona, mediante un Sistema Multi-Sonda, YSI-556-M. El valor total promedio de producción de hojarasca, según el área de cada zona, fue de $19.12 \pm 1.23 \mathrm{gPS} / \mathrm{m}^{2}$.mes (2.29 t/ha.año). Esta baja productividad se atribuye al clima seco de la región y a la poca amplitud de las mareas. Avicennia germinans y Laguncularia racemosa produjeron poco más del 80 $\%$ de toda la hojarasca, mientras que Rhizophora mangle sólo contribuyó con el $16 \%$ y Conocarpus erectus $<4 \%$. Existe una correlación significativa entre las variables abióticas del sustrato (principalmente la salinidad y la profundidad del agua intersticial) y la cantidad de hojarasca producida. Se concluye que, existen variaciones espaciales significativas en las variables abióticas del sustrato que se correlacionan con diferencias en la composición por especies del manglar y que, junto con las etapas de los ciclos vitales de esas especies, generan variaciones significativas en la cantidad y composición de la hojarasca producida. La investigación futura se enfocará en la cuantificación de las variaciones espaciales de la estructura del bosque y su relación con la producción de hojarasca.

Palabras clave: caída de hojarasca, manglar, humedales costeros, México, Pacífico oriental tropical.

\section{REFERENCIAS}

Aburto-Oropeza, O., Ezcurra, E., Danemann, G., Valdez, V., Murray, J., \& Sala, E. (2008). Mangroves in the Gulf of California increase fishery yields. Proceedings of the National Academy of Sciences, 105(30), 10456-10459.

Adame, M. F., Jimenez, A. Z., Teutli, C., Caamal, J. P., Andueza, M. T., Adame, H. L., Cano, R., Arana, H. H., Torres-Lara, R., \& Herrera Silveira, J. (2013). Drivers of Mangrove Litterfall within a Karstic Region Affected by Frequent Hurricanes. Biotropica, 45(2), 147-154.

Agraz-Hernández, C. M., García-Zaragoza, C., IriarteVivar, S., Flores-Verdugo, F. J., \& Moreno-Casasola, P. (2011). Forest structure, productivity and species phenology of mangroves in the La Mancha lagoon in the Atlantic coast of Mexico. Wetlands Ecology and Management, 19(3), 273-293.

Aké-Castillo, J. A., Vézquez, G., \& López-Portillo, J. (2006). Litterfall and decomposition of Rhizophora mangle L. in a coastal lagoon in the southern Gulf of Mexico. Hydrobiologia, 559(1), 101-111. 
Alongi, D. M. (2002). Present state and future of the world's mangrove forests. Environmental Conservation, 29(3), 331-349.

Alongi, D. M., Clough, B. F., \& Robertson, A. I. (2005). Nutrient-use efficiency in arid-zone forests of the mangroves Rhizophora stylosa and Avicennia marina. Aquatic Botany, 82, 121-131.

Anónimo. (1958). Symposium on the classification of brackish waters. Venice, April 8-14, 1958. Archives for Limnology and Oceanography, 11(suppl.), 1-248.

Arreola-Lizárraga, J. A., Flores-Verdugo, F. J., \& OrtegaRubio, A. (2004). Structure and litterfall of an arid mangrove stand on the Gulf of California, Mexico. Aquatic Botany, 79, 137-143.

Barreiro-Güemes, M. T. (1999). Aporte de hojarasca y renovación foliar del manglar en un sistema estuarino del Sureste de México. Revista de Biología Tropical, 47(4), 729-737.

Bouillon, S., Borges, A. V., Castañeda-Moya, E., Diele, K., Dittmar, T., Duke, N. C., Kristensen, E., Lee, S. Y., Marchand, C., Middelburg, J. J., Rivera-Monroy, V. H., Smith III, T. J., \& Twilley, R. R. (2008). Mangrove production and carbon sinks: A revision of global budget estimates. Global Biogeochemical Cycles, 22, GB2013.

Bunt, J. S. (1995). Continental scale patterns in mangrove litter fall. Hydrobiologia, 295(1-3), 135-140.

Comisión Nacional para el Conocimiento y Uso de la Biodiversidad (CONABIO). (2009). Sitios de manglar con relevancia biológica y con necesidades de rehabilitación ecológica. CONABIO, México D.F. http://www.biodiversidad.gob.mx/ecosistemas/manglares2013/sitiosPrioritarios.htm

Coronado-Molina, C., Alvarez-Guillén, H., Day Jr, J. W. Reyes, E., Perez, B. C., Vera-Herrera, F., \& Twilley, R. (2012). Litterfall dynamics in carbonate and deltaic mangrove ecosystems in the Gulf of Mexico. Wetlands Ecology and Management, 20(2), 123-136.

Day, J. W., Coronado-Molina, C., Vera-Herrera, F. R., Twilley, R., Rivera-Monroy, V. H., Alvarez-Guillén, H., Day, R., \& Conner, W. (1996). A 7 year record of above-ground net primary production in a southeastern Mexican mangrove. Aquatic Botany, 55(1), 39-60.

Flores-Verdugo, F., González-Farías, F., Ramírez-Flores, O., Amezcua-Linares, F., Yáñez-Arancibia, A., Alvarez-Rubio, M., \& Day, J. W. (1990). Mangrove ecology, aquatic primary productivity, and fish community dynamics in the Teacapán-Agua Brava lagoon-estuarine system (Mexican Pacific). Estuaries, 13(2), 219-230.

Flores-Verdugo, F., González-Farías, F., Zamorano, D. S., \& Ramírez-García, P. (1992). Mangrove ecosystems of the Pacific coast of Mexico: distribution, structure, litterfall and detritus dynamics. Coastal plant communities of Latin America, 17, 269-288.

González-Sansón, G., Aguilar-Betancourt, C., KosonoyAceves, D., Lucano-Ramírez, G., Ruiz-Ramírez, S., Flores-Ortega, J. R., Hinojosa-Larios, A., \& SilvaBátiz, F. (2014). Composición por especies y tallas de los peces en la laguna Barra de Navidad, Pacífico central mexicano. Revista de Biología Tropical, 61(1), 129-144.

Kristensen, E., Bouillon, S., Dittmar, T., \& Marchand, C. (2008). Organic carbon dynamics in mangrove ecosystems: a review. Aquatic Botany, 89(2), 201-219.

Lanza, G. de la (1994). Química. En G. de la Lanza \& C. Cáceres (Eds.), Lagunas costeras y el litoral mexicano (pp. 127-198). Universidad Autónoma de Baja California Sur.

Leach, G. J., \& Burgin, S. (1985). Litter production and seasonality of mangroves in Papua New Guinea. Aquatic Botany, 23(3), 215-224.

Legendre, P., \& Gallagher, E. D. (2001). Ecologically meaningful transformations for ordination of species data. Oecologia, 129(2), 271-280.

Legendre, P., \& Legendre, L. (2012). Numerical Ecology. Amsterdam: Elsevier.

López-Medellín, X., \& Ezcurra, E. (2012). The productivity of mangroves in northwestern Mexico: a meta-analysis of current data. Journal of Coastal Conservation, 16(3), 399-403.

López-Portillo, J. L. \& Ezcurra, E. (1985) Litter fall of Avicennia germinans L. in a one year cycle in a mudflat at the laguna de Mecoacan, Tabasco, Mexico. Biotropica, 17(3), 186-190.

Lugo, A. E., \& Snedaker, S. C. (1974). The ecology of mangroves. Annual Review of Ecology and Systematics, 39-64.

Mann, K., \& Lazier, J. (2006). Dynamics of Marine Ecosystems. Biological-Physical Interactions in the Oceans. Malden: Blackwell.

Oksanen, J., Guillaume Blanchet, F., Kindt, R., Legendre, P., Minchin, P. R., O’Hara, R. B., Simpson, G. L., Solymos, P., Henry, M., Stevens, H., \& Wagner, H., (2013). Vegan: Community Ecology Package. R package version 2.0-10. http://CRAN.R-project.org/ package $=$ vegan

Quinn, G. P., \& Keugh, M. J. (2002). Experimental design and Data Analysis for Biologists. Cambridge: Cambridge University Press.

Rodríguez-Zúñiga, M. T., Troche-Souza, C., Vázquez-Lule, A. D., Márquez-Mendoza, J. D., Vázquez-Balderas, B., Valderrama-Landeros, L., Velázquez-Salazar, S., Cruz-López, M. I., Ressl, R., Uribe-Martínez, A., Cerdeira-Estrada, S., Acosta-Velázquez, J., DíazGallegos, J., Jiménez-Rosenberg, R., Fueyo-Mac 
Donald, L., \& Galindo-Leal, C. (2013). Manglares de México/Extensión, distribución y monitoreo. Comisión Nacional para el Conocimiento y Uso de la Biodiversidad. México D.F.

Rzedowski, J. (2006). Vegetación de México. 1ra. Edición Digital, Comisión Nacional para el Conocimiento y Uso de la Biodiversidad, México.

Saenger, P., \& Snedaker, S. C. (1993). Pantropical trends in mangrove above-ground biomass and annual litterfall. Oecologia, 96(3), 293-299.

Silva-Bátiz, F. de A., González-Sansón, G., Nené, A., Godínez, E., Franco, M. del C., Corgos, A., Hernández, S., Hinojosa, J. A., Galván, V. H., \& Rojo, J. (2012). Bases para el manejo y conservación de la laguna de Barra de Navidad, Jalisco. Guadalajara: Editorial Página Seis.
StatSoft (2006). STATISTICA (data analysis software system) version 7.1. www.statsoft.com.

ter Braak, C. J. F., \& Smilauer, P. (2002). Canoco reference manual and CanoDraw for Windows user_s guide: software for canonical community ordination. Version 4.5. Microcomputer power, Ithaca, New York, USA.

Underwood, A. (1997). Experiments in Ecology: Their Logical Design and Interpretation Using Analysis of Variance. Cambridge: Cambridge University Press.

Utrera, M. E., \& Moreno, P. (2008). Mangrove litter dynamics in La Mancha lagoon, Veracruz, México. Wetlands Ecology and Management, 16(1), 11-22.

Zar, J. H. (2010). Biostatistical Analysis. New Jersey: Prentice-Hall. 
